\title{
Reducing Anxiety for Dental Visits
}

\author{
Kathryn Elizabeth Jones ${ }^{[0000-0001-7939-9314]}$, Fernando \\ Loizides ${ }^{[0000-0003-0531-6760]}$, Parisa Eslambolchilar ${ }^{[0000-0003-4610-1643]}$, Ilona \\ Johnson, Shannu Bhatia, Owen Crawford, McClaine Beirne, Raj Chand, Laura \\ Vuilleumier, and Idunah Araneta \\ Cardiff University, UK \\ \{jonesk90,floizides, eslambolchilarp, johnsonig, bhatiask, \\ crawford02, beirnem, chandr, vuilleumierl, aranetaij\}@cardiff .ac.uk
}

\begin{abstract}
This work addresses children's (under 6 years of age) fear and apprehension to visit dental clinics. We present a bespoke interactive Virtual Reality reproduction of the physical dental clinic, augmented with virtual characters and enriched with gamification style information for a richer user experience. The experience allows the user to navigate and familiarise themselves with the location and the procedures they will undertake before visiting the clinic. The experience is now being piloted at the Dental Public Health at the University Hospital of Wales.
\end{abstract}

Keywords: Virtual Reality · Dental Fear · Virtual Tour · Children

\section{Introduction and Motivation}

Children and parents are often anxious about dental treatment. Children are often reluctant to visit the dentist or a dental clinic due to dental fear. "Dental fear is defined as a specific anxiety, which is the predisposition for a negative experience in the dental surgery" $[2,5]$. There are many theories that give reasons and measure the anxiety, and fear, of children in this setting, such as their environment [4] and their perceptions of their dental staff [3].

Familiarisation with the dental setting, in advance of dental visits, can help to reduce anxiety [1]. This is particularly useful for children who often find the unfamiliar sights, sounds and equipment inside dental clinics unsettling. Virtual tours [6] and gamification [7] have been shown to successfully improve experiences for children undergoing operations under general anaesthetic.

In this paper, we present a fully functional prototype using Virtual Reality to help children experience their visit in advance and attempt to make them feel more at ease before a visit. The environment uses friendly digital characters and cues to make the experience more pleasant. Real $360^{\circ}$ footage from the dental clinic creates a reproduction of the actual environment that the children can explore to demystify the visit and increase confidence levels. The prototype system creates a Virtual Reality Environment which is interactive with a user. It currently has two main functions: to reduce patient anxiety and to provide education on dental hygiene and prevention. Firstly, younger patients often miss 
their dentist appointments due to fear of the unknown and lack of understanding of what they can expect from the environment and treatment when they get there. Therefore the prototype looks to simulate a dental appointment experience through a Virtual Reality tour. The dental patient will be able to navigate through the process at their own pace in order to familiarise themselves with the experience. Ultimately, this will significantly reduce their fear of the dentist as they gradually progress through their virtual appointment and become more familiar with what to expect when they arrive in the real world at the dental hospital. As a side effect it is hoped that reducing anxiety in young patients and making them more comfortable before coming to their appointment will reduce the DNA (Did Not Attend) rates at the hospital [8]. Secondly, within the Virtual Reality Environment there are opportunities to directly and passively communicate dental hygiene and prevention messages to patients through use of messages, games and quizzes.

\section{Artefact Description}

Using a user-centred design approach, we interviewed three dental practitioners to elicit requirements. We then created functional and non-functional requirements as well as personas. In order to begin phase one of the prototyping stages, we selected one of the three developed personas to focus on. The selected persona, a 6 year old girl called Evie, who had no disabilities, but her previous experience of dental appointments at her local dentistry practice meant she was already uncomfortable with the experience (creating a negative bias before visiting the dental hospital). Further to this Evie was generally uncomfortable with unfamiliar environments. Like most of the children her age, Evie is familiar with small screen display technologies, like mobile phones and tablets, and likes to play games using these devices.

Using the information relating to the identified stakeholders and requirements, Storyboards were created to help clarify the true goal of the application. Structured Dialogue Design (SDD) was used to for initial planning of the project because it gave the opportunity to hear the teams varying opinions without judgement. Following this a more detailed literature review was conducted, specifically looking at the user age and accessibility considerations, to support and enhance the design decisions being made. Based on the requirements Unified Modelling Language (UML) diagrams were made to consolidate ideas on what entities (Actors) would be interacting with the application and identify the core functionality that would be implemented.

Low-fidelity prototypes were developed as fully clickable mobile and desktop wire-frames in Axure. This allowed the whole team to brainstorm ideas of how the user would interact with the application. With regards to the web application wire-frames, the users journey when using the application was the focus, including aspects such as how many clicks does it take to complete the journey, is it obvious to the user where to click next, is the text big and simple enough to read. Initially we created wire-frames for both mobile and desktop devices 
with the intention to make this tour optimised for mobile, either as a flat screen tour or as a Virtual Reality tour using Google Cardboard ${ }^{1}$. The web application would guide the parent through some simple questions in order to discern which type of tour to display.

Feedback from the stakeholders, as well as our own thoughts relating to design improvements were used to move from low-fidelity to high-fidelity prototype. Our bespoke high-fidelity prototype aims to reduce the anxiety by giving the child a way to view the exact route they will take, and by explaining objects and events that will happen during their visit, as well as being an educational tool for dental hygiene. This has been achieved in the form of a $360^{\circ}$ Virtual Reality tour, which is designed to be viewed on desktop web browsers. Real $360^{\circ}$ images were taken of the dental clinic and then stitched to produce the Virtual Reality Environment.

The high-fidelity fully functional prototype was built with Unity (version 2017.4.10) ${ }^{2}$, making it easily customisable to a Head Mounted Display (HMD) or desktop experience. A web application was developed to host our tour involving a simple landing page with a brief description and guide, with links to a more in depth help page, and the tour itself. Built using React ${ }^{3}$ and bootstrapped using create-react-app ${ }^{4}$. The use of WebGL library provides a method of hosting a unity project within a web page with Blender ${ }^{5}$ being used to create $3 \mathrm{D}$ objects such as arrows and signs for use within the tour.

Care was also taken to produce appropriate interactions. Each interactive object can have an associated spoken element and some examples have been included in the prototype. This involved taking the full journey through the tour and deciding what a bunny would say at each point. This included interactions with a bunny (See Figure 1), icons, menus and games (See Figure 2). A fader was included to gently tele-port the user from one room to the other without being too abrupt, with the aim of maintaining a calm Virtual Reality Environment for the children.

Collaboration, co-production and inter-professional working provided a different view of the dental setting enabling the creation of Virtual Reality Environments designed to familiarise children and their parents with the dental setting. The prototype will be of use for developing a production ready application for children and parents visiting the dentist. It will also support dental student learning by providing an opportunity to view the dental environment from the point of view of a child, parent and someone unfamiliar with the dental environment and contribute information to the development of a final single application. An evaluation of prototype application is being undertaken to identify

\footnotetext{
${ }^{1}$ https://vr.google.com/cardboard/ - Accessed March 2019

2 https://unity.com/ - Accessed March 2019

${ }^{3}$ https://reactjs.org/ - Accessed March 2019

${ }^{4}$ https://developer.mozilla.org/en-US/docs/Web/API/WebGL_API - Accessed April 2019

${ }^{5}$ https://www.blender.org/ - Accessed April 2019
} 


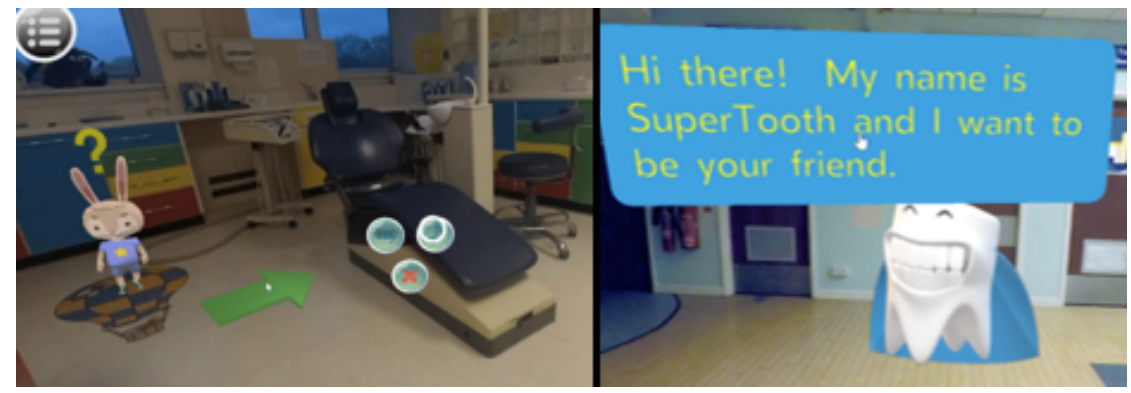

Fig. 1. The Virtual Reality Environment - exact replica of the dental clinic - with interactive characters to guide children.

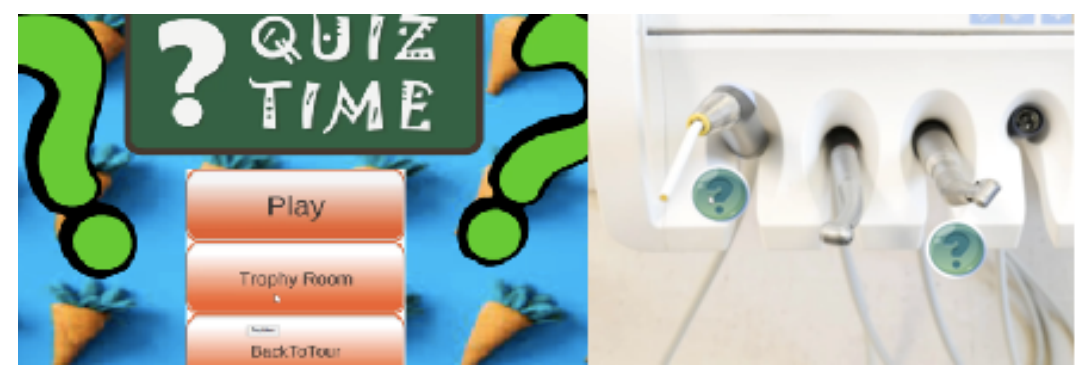

Fig. 2. Interactive scenes: Left: Quiz to learn about tooth hygiene, Right: information on the tools the child will be seeing and will be used for treatment

the most useful features to include in a single application for use by children and their parents.

\section{References}

1. Pharmacological behaviour management clinical guidelines. https: //www.rcseng.ac.uk/library-and-publications/rcs-publications/docs/ non-pharmacological-behaviour-management, 2019.

2. IH Aartman, J Hoogstraten, AH Schuurs, et al. Self-report measurements of dental anxiety and fear in children: a critical assessment. ASDC journal of dentistry for children, 65(4):252-8, 1998.

3. Maha Alsarheed. Childrens perception of their dentists. European journal of dentistry, 5(2):186, 2011.

4. Goran Koch, Sven Poulsen, Ivar Espelid, and Dorte Haubek. Pediatric dentistry: a clinical approach. John Wiley \& Sons, 2017.

5. Sunil Raj, Manisha Agarwal, Kiran Aradhya, Sapna Konde, and V Nagakishore. Evaluation of dental fear in children during dental visit using children's fear survey schedule-dental subscale. International journal of clinical pediatric dentistry, 6(1):12, 2013. 
6. Jung-Hee Ryu, Ah-Young Oh, Hee-Jeong Yoo, Jin-Hee Kim, Jin-Woo Park, and Sung-Hee Han. The effect of an immersive virtual reality tour of the operating theater on emergence delirium in children undergoing general anesthesia: A randomized controlled trial. Pediatric Anesthesia, 29(1):98-105, 2019.

7. Jung-Hee Ryu, Jin-Woo Park, Francis Nahm, Young-Tae Jeon, Ah-Young Oh, Hak Lee, Jin-Hee Kim, and Sung-Hee Han. The effect of gamification through a virtual reality on preoperative anxiety in pediatric patients undergoing general anesthesia: A prospective, randomized, and controlled trial. Journal of clinical medicine, 7(9):284, 2018.

8. Pia Wogelius and Sven Poulsen. Associations between dental anxiety, dental treatment due to toothache, and missed dental appointments among six to eight-year-old danish children: a cross-sectional study. Acta Odontologica Scandinavica, 63(3):179$182,2005$. 